\title{
Síndrome de ojo seco asociado a fármacos sistémicos
}

\section{Dry eye syndrome associated with systemic drugs}

\author{
Jesús Guerrero Becerril," Enrique O Graue Hernández*
}

Citar como: Guerrero BJ, Graue HEO. Síndrome de ojo seco asociado a fármacos sistémicos. An Med (Mex). 2020 ; 65 (4): $275-279$. https://dx.doi.org/10.35366/97465

\section{RESUMEN}

El síndrome de ojo seco es un motivo muy frecuente de consulta oftalmológica, caracterizado por un aumento de la osmolaridad en lágrima, inestabilidad de la película lagrimal e inflamación de la superficie ocular. Las principales causas son: edad, género femenino, disfunción de glándulas de Meibomio, uso de lentes de contacto, factores ambientales y medicamentos sistémicos. Los principales mecanismos involucrados en la génesis del síndrome ojo seco incluyen la disminución en el componente acuoso de la lágrima, alteración en la innervación corneal y la toxicidad directa a las glándulas lagrimales principales y accesorias. Muchos son los fármacos que se relacionan con ojo seco que por la frecuencia en su uso cotidiano son importantes en la práctica de la medicina general. Algunos de los grupos involucrados son: los antiinflamatorios no esteroideos, medicamentos para enfermedad ácido-péptica, sulfonilureas, antihipertensivos, ansiolíticos, antidepresivos, antipsicóticos, esteroides sistémicos, anticonceptivos orales, antihistamínicos y fármacos para tratar la enfermedad de Parkinson, entre otros. Esta investigación tiene como objetivo revisar brevemente los medicamentos sistémicos y los posibles mecanismos por los que se relacionan con ojo seco.

Palabras clave: Ojo seco, fármacos, córnea, enfermedad sistémica.

Nivel de evidencia: III

* Instituto de Oftalmología Fundación Conde de Valenciana FAP, Córnea, Enfermedades Externas y Cirugía Refractiva. Ciudad de México, México.

Recibido para publicación: 03/09/2020. Aceptado: 19/11/2020.

Correspondencia: Dr. Enrique 0 Graue Hernández Instituto de Oftalmología Fundación Conde de Valenciana FAP.

Chimalpopoca Núm. 14, Col. Obrera, 06800,

Cuauhtémoc, Ciudad de México, México.

Teléfono: 55 5442-1700; Fax: 55 5578-9748

E-mail: egraueh@gmail.com

\begin{abstract}
Dry eye syndrome is a very common cause of ophthalmological consultation, it is a condition characterized by high tear osmolarity, tear film instability and ocular surface inflammation. The main etiologies are age, feminine gender, meibomian gland dysfunction, contact lens use, environmental factors and systemic drugs. The main mechanisms that cause dry eye are reduction in tear production, alteration of the corneal nerves or a direct toxicity to the lacrimal gland. Many drugs are related to dry eye syndrome, this are important in daily medical practice due to the frequent use. Some of the group of drugs that have been related to dry eye syndrome are: NSAIDs (nonsteroidal anti-inflammatory drugs), gastric protector drugs, antihypertensive agents, anxiolytics, oral contraceptives, antidepressants, antipsychotics, antihistamines and drugs for treating Parkinson's disease, among others. The purpose of this review is to briefly summarize the most common systemic medications and the possible mechanism related to dry eye disease.
\end{abstract}

Keywords: Dry eye syndrome, drugs, cornea, systemic disease.

Level of evidence: III

El síndrome de ojo seco afecta a cientos de millones de personas en todo el mundo y es una de las causas más frecuentes de consulta oftalmológica. Es un círculo vicioso caracterizado por inestabilidad de la película lagrimal, osmolaridad incrementada e inflamación de la superficie ocular. Por lo general, resulta en síntomas como malestar ocular, alteraciones visuales 
y un potencial daño a la superficie ocular. El número cada vez mayor de casos con esta patología es un testimonio importante de que esta entidad necesita atención inmediata y comprensión de la fisiopatología subyacente e identificación de los factores que pueden ocasionar ojo seco. ${ }^{1,2}$

El ojo seco afecta la productividad en el lugar de trabajo, limitando las actividades necesarias como leer o utilizar una computadora por largos periodos de tiempo. En Estados Unidos, este problema conlleva una carga económica de aproximadamente $3.8 \mathrm{mil}$ millones de dólares por gastos de salud al año. ${ }^{3}$

El síndrome de ojo seco tiene muchas causas, las cuales frecuentemente se superponen e interactúan. Suele coexistir con otras condiciones o puede ser exacerbado por distintos factores ambientales. ${ }^{3}$ Las dos categorías predominantes y mutuamente no excluyentes son ojo seco acuodeficiente y ojo seco evaporativo. Existe cada vez más evidencia de que ambos tipos de ojo seco pueden coexistir e interactuar entre ellos.

La prevalencia aproximada de síndrome de ojo seco va del 5-50\%, aunque basándose sólo en signos clínicos llega a ser hasta de $75 \%$ en algunas poblaciones. Las mujeres son más afectadas que los hombres y la presencia de signos y síntomas es directamente proporcional a la edad. ${ }^{1}$

El diagnóstico de ojo seco es complicado debido a la importante inconsistencia entre los síntomas reportados por el individuo y los signos observados. Esta inconsistencia se puede explicar por la variabilidad de los resultados de los exámenes utilizados para este padecimiento, la variabilidad del proceso de la enfermedad, la subjetividad de los síntomas, variabilidad individual del umbral del dolor y las respuestas cognitivas a preguntas acerca de sensibilidad ocular. ${ }^{3}$

Los factores de riesgo se clasificaron en el TFOS DEWS II como consistente, probable y como no concluyente. La edad, género, etnia, disfunción de glándulas de Meibomio (DGM), síndrome de Sjögren, deficiencia de andrógenos, uso de computadora, uso de lentes de contacto, terapia de reemplazo hormonal, trasplante de células madre hematopoyéticas, algunos factores del medio ambiente (lugares con poca humedad, contaminación) y medicamentos sistémicos (antihistamínicos, antidepresivos, ansiolíticos e isotretinoína) se identificaron como factores consistentes. ${ }^{1}$

\section{OJO SECO Y FÁRMACOS SISTÉMICOS}

En Estados Unidos, cuatro de cada cinco adultos utilizan medicamentos recetados por su médico, medicamentos de venta libre o suplementos nutri- cionales en una semana. ${ }^{3,4}$ Debido a que el uso de fármacos y de productos a base de hierbas es más común en población geriátrica debido a la mayor prevalencia de padecimientos crónico-degenerativos, el conocimiento de estos productos específicos es importante, ya que juegan un papel vital en la salud ocular de estos casos..$^{3-5}$

De acuerdo con la clasificación de ojo seco iatrogénico, los medicamentos sistémicos se clasificaron en el apartado 1A (inducidos por fármacos sistémicos). Entre los 100 medicamentos más vendidos en Estados Unidos, por lo menos 22 ocasionan ojo seco, de los nueve fármacos conocidos por tener concentraciones en lágrima, ocho ocasionan ojo seco. La mayoría de los estudios que analizan ojo seco inducido por fármacos sólo incluyen familias de fármacos y no de manera individual. ${ }^{6}$

Los mecanismos por los cuales se puede generar un ojo seco iatrogénico secundario a fármacos son los siguientes: disminución en la producción de lágrima, alteración en los nervios aferentes y secreción refleja, efectos inflamatorios en las glándulas o toxicidad directa a través de la lágrima. El $62 \%$ de los casos de ojo seco en pacientes de la tercera edad se ha relacionado con algún medicamento sistémico, en especial: antiinflamatorios no esteroideos (AINE; OR 1.30), diuréticos (p. ej: furosemide, OR 1.25), vasodilatadores (OR 1.37), analgésicos/antipiréticos (OR 1.28), fármacos protectores gástricos (p. ej: ranitidina, OR 1.44), sulfonilureas (OR 1.3), glucósidos cardiacos (OR 1.28), betabloqueadores (p. ej: propanolol), ansiolíticos/benzodiacepinas (p. ej: lorazepam, OR 2.35), antimicrobianos (OR 1.88), antidepresivos/ antipsicóticos (p. ej: amitriptilina, tioridazina, OR 2.54), anticonvulsivantes (p. ej: ácido valproico), hipotensores (p. ej: candesartán, OR 1.98), esteroides inhalados (OR 2.04), esteroides sistémicos (OR 1.60), anticonceptivos orales, terapia de reemplazo hormonal (OR 1.60), fármacos para hiperplasia prostática benigna (OR 1.35), multivitamínicos (OR 1.41), descongestivos (p. ej: pseudoefedrina), fármacos para tratamiento de enfermedad de Parkinson (p. ej: trihexifenidilo), algunos suplementos herbales (p. ej: echinacea, niacin y kava) y antihistamínicos (p. ej: cetirizina, OR 1.67). ${ }^{2,3,6}$ En la Tabla 1 se pueden observar las principales familias de fármacos involucradas con la patogénesis de ojo seco y algunos ejemplos.

\section{MECANISMOS DE OJO SECO}

Un grupo de fármacos que pueden ocasionar ojo seco son aquéllos con actividad anticolinérgica, en 
Tabla 1: Principales familias de fármacos y ejemplos asociados al síndrome de ojo seco (se muestran los fármacos más empleados en nuestro medio).

\begin{tabular}{|c|c|}
\hline Familia de fármaco & Ejemplos \\
\hline Antihistamínicos y descongestivos & Cetirizina, loratadina, fexofenadina, clorfeniramina, dexclorfeniramina, fenilefrina, pseudoefedrina \\
\hline Antidepresivos & $\begin{array}{l}\text { Amitriptilina, nortriptilina, clomipramina, imipramina, fluoxetina, citalopram, escitalopram, paroxetina, } \\
\text { sertralina, duloxetina, venlafaxina, mirtazapina, bupropión, trazodona }\end{array}$ \\
\hline Anticonvulsivos & Ácido valproico, lamotrigina, fenobarbital \\
\hline Antisicóticos & Tioridazina, clozapina, clorpromazina \\
\hline Antiespasmódicos & Metocarbamol \\
\hline Agentes protectores gástricos & Cimetidina, ranitidina \\
\hline Fármacos respiratorios sistémicos & Bromuro de ipratropio, tiotropio \\
\hline AINE & Ibuprofeno, ketoprofeno, ácido acetil salicílico, diclofenaco \\
\hline Opiáceos & Dextropropoxifeno, morfina \\
\hline Antiarrítmicos & Amiodarona, quinidina, flecainida, mexiletina \\
\hline Agentes antivirales & Aciclovir \\
\hline Antimaláricos & Hidroxicloroquina \\
\hline Incontinencia urinaria & Tolterodina \\
\hline Productos herbales & $\begin{array}{l}\text { Niacina, echinacea, kava, belladona, Datura stramonium, Hyoscyamus niger, Mandragora } \\
\text { officinarum, Scopolia }\end{array}$ \\
\hline
\end{tabular}

específico los asociados con proteína G, éstos pueden afectar los acinos de la glándula lagrimal y a las células productoras de moco, lo que reduce el componente acuoso y mucinoso y altera la estabilidad de la lágrima. Como efecto periférico, los antihistamínicos pueden producir sequedad en la mucosa oral y respiratoria. ${ }^{2}$ Asimismo, se han identificado receptores colinérgicos en las células epiteliales de las glándulas de Meibomio, posiblemente afectando la calidad de la lágrima en este componente. Además, los fármacos adrenérgicos (betabloqueadores y alfa-agonistas) alteran tanto la producción como la calidad de la lágrima, este mecanismo se cree que es secundario a la producción de proteína cinasa $\mathrm{C}$ y a la concentración de calcio intracelular. ${ }^{6}$ Los betabloqueadores reducen los niveles de lisozima y de inmunoglobulina $\mathrm{A}$, en adición a esto pueden causar anestesia corneal y disminución en el tiempo de ruptura lagrimal (TRL). ${ }^{2}$

Los cuatro antiinflamatorios no esteroideos (AINE) que se han visto más relacionados con el desarrollo de ojo seco son ibuprofeno, ketoprofeno, diclofenaco y ácido acetilsalicílico, este último alcanza concentraciones importantes en lágrima y ocasiona ojo seco y queratitis punteada superficial. ${ }^{2}$ Ketoprofeno raras veces ocasiona ojo seco per se, pero sí tiene el potencial de agravar síntomas preexistentes.
Algunos agentes quimioterapéuticos como metotrexate, mitomicina $\mathrm{C}$ y busulfán han mostrado causar alteraciones en la calidad de la lágrima, así como en la secreción refleja. ${ }^{6}$

Ácido retinoico en exceso ocasiona blefaritis y ojo seco, el mecanismo propuesto es atrofia de las glándulas de Meibomio y después alteración en la secreción de lípidos.

La isotretinoína impide la adecuada función de las glándulas de Meibomio, incrementando la evaporación de la lágrima. ${ }^{3}$

Los antidepresivos tienen una estructura química similar a la atropina, un agente anticolinérgico con efectos antimuscarínicos bien conocidos. El efecto antagonista de los antidepresivos en los receptores muscarínicos ocasiona ojo seco al afectar el músculo liso y el tejido glandular. ${ }^{7}$

Los anticonvulsivos, al tener un efecto anticolinérgico, bloquean el efecto del neurotransmisor acetilcolina. Algunos agentes específicos que han sido reportados son: fenobarbital, primidona, ácido valproico y lamotrigina. ${ }^{8}$

Los efectos conocidos de los antipsicóticos son: disminución de la calidad visual, disminución en la acomodación, cambios en la sensibilidad al color, fotofobia, reacción alérgica y ojo seco. ${ }^{7,8}$ Los fármacos para tratar la enfermedad de Parkinson tienen un 
An Med (Mex) 2020; 65 (4): 275-279

mecanismo similar en causar ojo seco al de antidepresivos y antipsicóticos. Los más utilizados son: biperidina, benztropina, prociclidina, trihexifenidilo, levodopa, bornaprina y pramipexol. ${ }^{2}$

Las benzodiacepinas reducen la secreción salival y lagrimal al unirse a receptores muscarínicos, además del efecto de reacción cruzada que pueden tener esta familia de fármacos y ocasionar síndrome de Stevens-Johnson. ${ }^{6}$

Las mujeres menopáusicas y postmenopáusicas tienen más riesgo de tener ojo seco. ${ }^{9}$ Las hormonas sexuales tienen efectos muy importantes en la secreción de lágrima, función de las glándulas de Meibomio y densidad de las células caliciformes. La combinación de progesterona con estrógenos ha mostrado menor riesgo en ocasionar ojo seco que el uso de estrógenos solo. ${ }^{2}$

Algunos fármacos aislados que pueden ocasionar ojo seco son amiodarona y flecainida, donde se ha observado que el $10 \%$ de los casos puede presentar TRL disminuido. ${ }^{2,10}$

Los suplementos herbales y medicina complementaria cada vez empiezan a tomar más terreno, en Estados Unidos se calcula que alrededor de 15 millones de personas utilizan algún tipo de producto herbal o multivitamínico. ${ }^{11}$ La niacina (vitamina B3) ha sido utilizada para tratar anorexia nervosa, depresión, hipercolesterolemia, diabetes mellitus y migraña, ha sido relacionada con disminución de la visión, edema macular quístico y ojo seco. La echinacea es utilizada popularmente para el tratamiento y prevención de síntomas respiratorios y se ha demostrado ser causante de síntomas de ojo seco. La kava es utilizada hoy en día para el tratamiento del trastorno de ansiedad generalizado, síntomas de climaterio también han sido identificados como un agente causal de ojo seco. ${ }^{2,12}$

La polifarmacia (uso de cinco fármacos o más) aumenta el riesgo relativo de presentar un ojo seco iatrogénico, este mecanismo es complejo; sin embargo, el mecanismo exacto no se conoce. ${ }^{6}$ En adición, las personas de la tercera edad pueden tener más factores de riesgo, además de que son más propensos a tomar varios fármacos. ${ }^{3}$

En la Figura 1 se muestra un caso representativo del Instituto de Oftalmología Fundación Conde de Valenciana.

\section{TRATAMIENTO Y RECOMENDACIONES}

El primer paso es identificar el fármaco responsable del cuadro clínico, se recomienda retirar el fármaco y realizar una prueba terapéutica e introducirlo de nuevo, observando la respuesta clínica. En algunos casos, esta modalidad no es posible, en éstos se puede correlacionar el inicio de síntomas con el inicio del fármaco. Asimismo, retirar el fármaco no siempre es recomendable, por lo que se debe modificar el fármaco por algún otro con mecanismo de acción alternativo y puede ayudar a mejorar los síntomas de ojo seco. Otra alternativa es reducir la dosis del fármaco, lo que permite mantener una buena acción terapéutica y reducir la sintomatología. En caso de que los síntomas no sean muy graves, siempre se puede optar por tratamiento tópico con lubricantes oculares o algún otro agente local. ${ }^{6}$

Lo ideal sería tener agentes terapéuticos con mayor afinidad por receptores específicos y reducir afinidad por los receptores no específicos. Otras medidas serían modificaciones químicas que reduzcan la permeabilidad a tejidos oculares, desarrollo de sistemas de liberación que podrían tener un efecto en la canti-
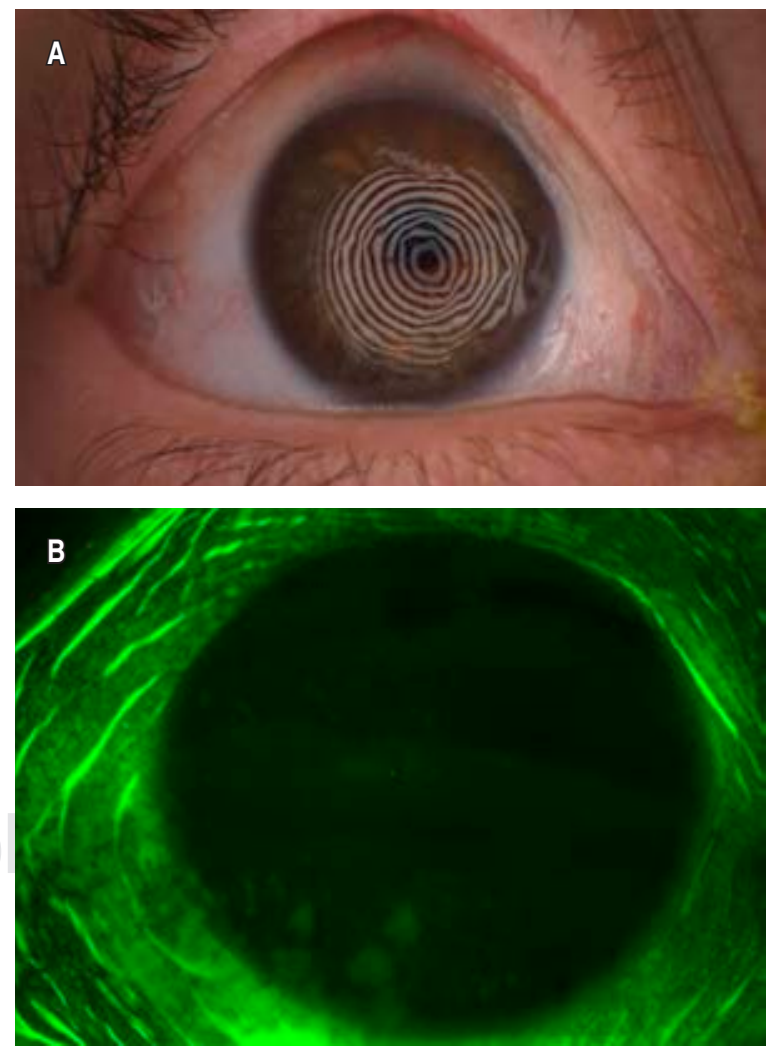

Figura 1: Imagen clínica obtenida por medio del topógrafo Keratograph $5 \mathrm{M}$ de OCULUS. A) Muestra una distorsión franca de los anillos reflejados en la córnea de una mujer de 46 años con diagnóstico de trastorno depresivo mayor en tratamiento con fluoxetina. B) Tinción con fluoresceína y queratitis punteada superficial importante en sector inferior y central. 
dad de fármaco que llega al ojo y, por último, cambios en la administración y dosificación que eviten una mayor concentración en tejidos oculares. ${ }^{6}$

\section{CONCLUSIÓN}

El síndrome de ojo seco puede ser causado por múltiples causas iatrogénicas; sin embargo, se necesitan estudios adicionales para evaluar el ojo seco iatrogénico, en particular el causado por fármacos sistémicos. Es necesaria una evaluación profunda y un interrogatorio acerca de los fármacos que consume el sujeto, así como del inicio de estos agentes sistémicos y su correlación apropiada con el inicio de los síntomas. Como médico no oftalmólogo, es de vital importancia mantener en mente los principales fármacos que pueden estar asociados a este síndrome para un manejo multidisciplinario y un pronto envío a un oftalmólogo para evitar las potenciales complicaciones.

\section{BIBLIOGRAFÍA}

1. Craig JP, Nelson JD, Azar DT, Belmonte C, Bron AJ, Chauhan SK et al. TFOS DEWS II report executive summary. Ocul Surf. 2017; 15 (4): 802-812.

2. Askeroglu U, Alleyne B, Guyuron B. Pharmaceutical and herbal products that may contribute to dry eyes. Plast Reconstr Surg. 2013; 131: 159-167.

3. Clayton JA. Dry eye. N Engl J Med. 2018; 378 (23): 22122223.
4. Burke JP. Book Review Preventing Medication Errors (Quality Chasm Series.) By the Committee on Identifying and Preventing Medication Errors and the Board on Health Care Services. Edited by Philip Aspden, Julie A. Wolcott, J. Lyle Bootman, and Linda R. Cronenwett. 463 pp. Washington, DC, National Academies Press, 2007. 54.95. 978-0-309-10147-9 Medication Errors Second edition. Edited by Michael R. Cohen. 680 pp., illustrated. Washington, DC, American Pharmacists Association, 2007. 89.95. 978-1-58212-092-8. N Engl J Med. 2007; 357: 624-625. doi: 10.1056/NEJMbkrev58255.

5. Blomquist PH. Ocular complications of systemic medications. Am J Med Sci. 2011; 342 (1): 62-69. doi: 10.1097/ MAJ.0b013e3181f06b21.

6. Gomes JAP, Azar DT, Baudouin C, Efron N, Hirayama M, Horwath-Winter J, et al. TFOS DEWS II iatrogenic report. Ocul Surf. 2017;15: 511-538.

7. Wong J, Lan W, Ong LM, Tong L. Non-hormonal systemic medications and dry eye. Ocul Surf. 2011; 9 (4): 212-226.

8. Marino D, Malandrini A, Rocchi R, Selvi E, Federico A. Transient "sicca syndrome" during phenobarbital treatment. J Neurol Sci. 2011;300: 164.

9. Lamberts DW, Foster CS, Perry HD. Schirmer test after topical anesthesia and the tear meniscus height in normal eyes. Arch Ophthalmol. 1979; 97 (6): 1082-1085.

10. Ikäheimo K, Kettunen R, Mäntyjärvi M. Visual functions and adverse ocular effects in patients with amiodarone medication. Acta Ophthalmol Scand. 2002; 80 (1): 59-63.

11. Eisenberg DM, Kessler RC, Foster C, Norlock FE, Calkins DR, Delbanco TL. Unconventional medicine in the United States. Prevalence, costs, and patterns of use. N Engl J Med. 1993; 328 (4): 246-252.

12. Logan JL, Ahmed J. Critical hypokalemic renal tubular acidosis due to Sjögren's syndrome: association with the purported immune stimulant echinacea. Clin Rheumatol. 2003; 22 (2): 158-1589. doi: 10.1007/s10067-002-0671-4. 